Orange Journal / Volumen 2 Número 3/ Enero - Junio 2020

DOI: https://doi.org/10.46502/issn.2710-995X/2020.3.02

\title{
Cuantificación de fenoles y flavonoides totales en un extracto blando de flores de Calendula officinalis Linn
}

Total phenols and flavonoids quantification in a soft extract of Calendula officinalis L. flowers

\section{Resumen}

La Calendula officinalis L. ocupa un lugar destacado en el tratamiento de múltiples afecciones, debido a sus propiedades farmacológicas, dentro de las que se incluyen antibacteriana, antiinflamatoria, cicatrizante, astringente, antiséptica, hipotensora, antiespasmódica, antibiótica, fungicida y antioxidante. Con el objetivo de cuantificar la proporción de compuestos fenólicos y flavonoides totales presentes en un extracto blando de flores de la planta, se elaboraron 5 lotes de extracto blando (EB) de Calendula officinalis L. La determinación cualitativa de fenoles totales y flavonoides reveló la presencia de estos compuestos en los extractos, lo que se confirmó con la determinación cuantitativa, revelando la presencia de un contenido de 9,65 a $11,31 \mathrm{mgAG} / 0,1 \mathrm{~g}$ y 1,51 a $2,17 \mathrm{mgQ} / 0,1 \mathrm{~g}$ de fenoles y flavonoides, respectivamente.

Palabras clave: Calendula officinalis L., extracto blando, fenoles, flavonoides.

\begin{abstract}
Calendula officinalis L. occupies a prominent place in the treatment of multiple conditions, due to its pharmacological properties, such as antibacterial, antiinflammatory, healing, astringent, antiseptic, hypotensive, antispasmodic, antibiotic, fungicide and antioxidant. In order to quantify the proportion of phenolic compounds and total flavonoids in a soft extract of the plant's flowers, five lots of soft extract (SE) of Calendula officinalis L. were prepared, to be used as active compound in formulations for stomatological uses. The qualitative determination of total phenols and flavonoids

${ }^{5}$ Licenciada en Ciencias Farmacéuticas. Centro de Toxicología y Biomedicina (TOXIMED), Santiago de Cuba.

6 Máster en Servicios Farmacéuticos. Departamento de Farmacia, Facultad de Ciencias Naturales y Exactas, Universidad de Oriente, Santiago de Cuba, Cuba.

${ }^{7}$ Doctora en Ciencias de la Salud. Departamento de Farmacia, Facultad de Ciencias Naturales y Exactas, Universidad de Oriente, Santiago de Cuba, Cuba.
\end{abstract}




\section{ORANGE JOURNAL}

revealed the presence of these compounds in the extracts, which was confirmed with the quantitative determination, revealing the presence of a content of 9.65 to $11.31 \mathrm{mgAG}$ $/ 0.1 \mathrm{~g}$ and 1.51 to $2.17 \mathrm{mgQ} / 0.1 \mathrm{~g}$ of phenols and flavonoids, respectively, validating the use of the extracts in the treatment of oral diseases.

Keywords: Calendula officinalis L., soft extract, phenols, flavonoids.

\section{Introducción}

Desde tiempos ancestrales se han utilizado las plantas como alimentos y medicina. Actualmente los productos naturales y sus derivados representan más del $50 \%$ de todos los fármacos de uso clínico, siendo considerada la medicina tradicional de gran interés para los consumidores. Es de gran importancia garantizar la eficacia, calidad y seguridad de estos productos, mediante la realización de nuevas investigaciones que garanticen la ampliación de estos conocimientos (Ferreira et al., 2010; WHO, 2013).

En el reino vegetal existe un grupo de metabolitos secundarios conocidos como compuestos fenólicos (taninos, flavonoides, etc.), a los que se le atribuyen propiedades farmacológicas de interés, dentro de las que se encuentran principalmente la antiinflamatoria, antiséptica, cicatrizante y antioxidante de la gran mayoría de las plantas (Rotta et al, 2017). No obstante, la calidad y cantidad producida de estos compuestos por las plantas medicinales está muy condicionada a factores bióticos y abióticos relativos a los lugares donde se cultivan y cosechan (Llauradó et al, 2020).

La Calendula officinalis L., ocupa un lugar destacado dentro de las plantas utilizadas con el fin de combatir diversas afecciones, debido a sus múltiples propiedades farmacológicas (Yassen et al, 2010). En Cuba es una de las plantas medicinales aprobadas por el Ministerio de Salud Pública (MINSAP), por lo que aparece reportada en el Formulario Nacional de Fitofármacos y Apifármacos (MINSAP, 2014); crece adecuadamente en condiciones de cultivo y sus flores cumplen con los requisitos establecidos por las farmacopeas internacionales para su uso como planta medicinal (Lastra y Piquet, 1999; Paolini et al, 2010).

Investigaciones realizadas de los extractos de esta planta y en particular de sus flores muestran un amplio espectro de acciones farmacológicas, entre las que sobresalen antibacteriana, antiinflamatoria, cicatrizante, astringente, antiséptica, hipotensora, antiespasmódica, antibiótica, fungicida y antioxidante. Además, se han reportado propiedades colagogas, hipolipemiantes, inmunoestimulantes, antigripales y antitumorales (Arora, Rani y Sharma, 2013; Ayuso, Torrent y López, 2004; Cong et al, 2017).

Según lo reportado en la literatura (Roig, 1991; Arora et al, 2013), las decocciones de las flores de caléndula tienen un amplio espectro en cuanto al tratamiento de diversas afecciones, entre las que podemos citar para la curación de las heridas, como colutorios en las estomatitis, y en la piorrea; en el tratamiento de la gastritis, de las úlceras, hepatitis y otras enfermedades gastrointestinales; en el tratamiento de la hipertensión, taquicardia y arritmia; en el tratamiento de diversas afecciones del sistema urinario, así como en 
enfermedades del sistema nervioso central y periférico. De la información etnobotánica precedente se infiere que los extractos acuosos de las flores de $C$. officinalis $\mathrm{L}$. presentan propiedades farmacológicas como: cicatrizante, antiinflamatorio, antibacteriano y tranquilizante, muchas de las cuales podrían estar relacionadas al contenido de compuestos fenólicos en su estructura. En tal sentido, Amić y colaboradores (2007) informan que muchos de los efectos terapéuticos atribuidos a la flor de caléndula derivan de la presencia de flavonoides, entre los cuales destaca la quercetina, perteneciente al grupo de los flavonoles, aportando actividad antiinflamatoria, antioxidante y antimicrobiana. Todas estas bondades han derivado en el hecho que las flores de Calendula officinalis L. se conviertan en una materia prima natural de gran interés para la industria farmacéutica y que el monitoreo de la producción de flavonoides y fenoles en las plantaciones locales, constituya una vía de control de la calidad de la misma, para garantizar la presencia de estos metabolitos en cantidades adecuadas y que no se vea afectada la eficacia del preparado fitoterapéutico (Muñoz, 2014).

Es por ello que la presente investigación se plantea como objetivo determinar la composición química cuantitativa de fenoles totales y flavonoides en el extracto blando de sus flores, corroborando su potencial para ser usado como principio activo en el desarrollo de formulaciones para uso estomatológico.

\section{Marco teórico}

En el reino vegetal se encuentran ampliamente distribuidos los polifenoles o compuestos fenólicos, siendo moléculas naturales del metabolismo secundario de las plantas que engloban más de 8000 compuestos distintos, constituyendo un amplio grupo de sustancias químicas, con diferentes estructuras, propiedades químicas y actividad biológica (Valencia et al, 2017). Estos compuestos son responsables de numerosas de las propiedades organolépticas de los vegetales, contribuyendo con el color, sabor amargo, astringencia y aroma; además presentan diferentes propiedades en el organismo humano, tales como: antioxidantes, antiinflamatorias, antitrombóticas, antimicrobianas, antialérgicas, antitumorales y antiasmáticas, entre otras (Pérez, 2003; Dominguez, 2009; Dominguez, 2012). Estas propiedades no están asociadas a un solo compuesto fenólico, sino al conjunto de ellos, entre los que se destacan los derivados de ácidos cinámicos, ácidos benzoicos, estilbenos y flavonoides (Dominguez, 2009).

Los taninos son compuestos fenólicos poliméricos que se unen a las proteínas, desnaturalizándolas. El nombre de tanino procede de la antigua práctica de utilizar extractos vegetales para convertir la piel animal en cuero (Avalos y Pérez, 2009). La acción que mejor los define es la astringente, por lo que se han usado tradicionalmente para la protección de superficies inflamadas de la boca y en el tratamiento del catarro, bronquitis, quemaduras, heridas (coagulante), hemorroides y diarreas, y como antídoto en las intoxicaciones por metales pesados (Ogunleye e Ibitoye, 2003; Rotta et al, 2017) .

Por otra parte, se encuentran los flavonoides, uno de los grupos más estudiados dentro de los compuestos fenólicos, debido a su distribución numerosa y a su actividad antioxidante elevada, siendo el subgrupo de los flavonoles el más estudiado. Su esqueleto carbonado contiene 15 carbonos ordenados en dos anillos aromáticos unidos por un puente de tres 


\section{ORANGE JOURNAL}

carbonos (heterociclo). Se clasifican en función del grado de oxidación del puente de tres carbonos, siendo los principales: antocianinas, flavonas, flavonoles (quercetina, la miricetina, kaempferol), isoflavonas, flavanoles (catequina y derivados) (HernándezRosas, et al, 2018).

La acción farmacológica de estos metabolitos es variada y extensa, bien conocidas son sus propiedades: antiinflamatoria, antianafiláctica, antitumoral, antidiarreica, expectorante, estomáquica, carminativa, colagoga, antihemorrágica, antigonadotrópica, inmunomoduladora, antiagregante plaquetaria, antiespasmódica, antihelmíntica, cardioprotectora, hipoglicemiante, antioxidante, hepatoprotectora, estrogénica, diurética y analgésica. Se destaca asimismo su actividad antibacteriana y la antifúngica (Escalona, 2005; Avalos y Pérez, 2009). En C. officinalis se han detectado la presencia de los flavonoides quercetina, isohamnetina, rutina y kaempferol (Neukirch, D'Ambrosio, Via y Guerriero, 2004; Muley, Khadabadi y Banarase, 2009).

Atendiendo a todo lo planteado anteriormente, la presencia de compuestos fenólicos en el extracto blando de flores de $C$. officinalis L. validará su uso como principio activo de una formulación para uso estomatológico.

\section{Metodología}

\section{Características generales de la investigación}

Se realizó un estudio experimental, transversal, prospectivo y de enfoque cuantitativo para determinar la composición cuantitativa de fenoles y flavonoides del extracto blando de flores de Calendula officinalis L. El estudio se desarrolló en el laboratorio MediCubaSuiza, del Departamento de Farmacia de la Universidad de Oriente, en el período comprendido de febrero a mayo de 2018.

\section{Diseño experimental}

Se elaboraron 5 lotes de extracto blando (EB) de Calendula officinalis L., con flores suministradas entre los meses de octubre de 2017 a febrero de 2018, por la finca de plantas medicinales "La Rosita" ubicada en el municipio Santiago de Cuba y certificada por el Laboratorio de Control de la Calidad de la Empresa de Farmacia y Óptica de la provincia Santiago de Cuba. Los extractos fluidos se obtuvieron por el método de repercolación con 4 extracciones descrito en la Norma Ramal de Salud Pública (NRSP) 311 de 1991. Por la concentración a vacío de los mismos en un sistema de evaporación al vacío (Heidolph G1) se obtuvieron los extractos blandos.

\section{Composición química cualitativa}

Se determinó la composición química cualitativa, a través de los ensayos de cloruro férrico para taninos y fenoles, y los de ácido sulfúrico concentrado, Shinoda, álcalis y Rosemheim para flavonoides, de acuerdo a la técnica descrita por Ochoa, López y Colombat en 1999. 


\section{Cuantificación de fenoles totales}

Para la cuantificación de fenoles totales se empleó el método de Folin Ciocalteau con modificaciones. En un volumétrico de $25 \mathrm{~mL}$ se añadieron $5 \mathrm{~mL}$ de la muestra (extracto blando de Calendula officinalis L. y $2 \mathrm{~mL}$ del reactivo de Folin Ciocalteau. Se agitó y se dejó reposar por $5 \mathrm{~min}$. Luego se añadió $1 \mathrm{~mL}$ de solución de carbonato de sodio al $20 \%$, se agitó y se enrasó volumétrico con agua purificada. Se dejó reposar una hora a resguardo de la luz y se realizaron mediciones contra blanco de reactivos a 760nm. La concentración de fenoles totales fue calculada con la curva de calibración usando ácido gálico (25-150 $\mu \mathrm{L}$ ) como estándar (ecuación 1: $\mathrm{y}=0.0892 \mathrm{x}-0.0293 ; \mathrm{R}^{2}=0,9948 ; \mathrm{F}=765,73 ; \mathrm{p}=0,0000$ y pintercepto $=0,0799$ ). Los resultados fueron expresados como miligramos equivalentes de ácido gálico por cada 0,1 gramos de extracto blando (mgAG/0,1g).

\section{$\underline{\text { Cuantificación de flavonoides }}$}

La cuantificación de flavonoides fue determinada a través del método colorimétrico con cloruro de aluminio en etanol absoluto a $415 \mathrm{~nm}$. Se tomó $0,1 \mathrm{~g}$ de extracto blando y se diluyó en $10 \mathrm{~mL}$ de etanol al $60 \%$. Se adicionó $1 \mathrm{~mL}$ de cloruro de aluminio al $2 \%$ en etanol absoluto. Se agitó evitando pérdida de la solución y se completó volumen con etanol al $60 \%$. Se midió la absorbancia a $415 \mathrm{~nm}$. La concentración de flavonoides fue calculada a través de la curva de calibración, utilizando quercetina $(25-125 \mu \mathrm{L})$ como patrón. Los resultados fueron expresados como miligramos equivalentes de quercetina por cada 0,1 gramos de extracto blando (mgQuerc/0,1g), obtenidos a través de la ecuación2: $\mathrm{y}=0,0726 x+0.0203\left(\mathrm{R}^{2}=0,9931 ; \mathrm{F}=434,03 ; \mathrm{p}=0,0002 ; \mathrm{p}\right.$-intercepto=0,1768).

\section{Análisis estadístico y procesamiento de la información}

Para el procesamiento matemático y análisis estadístico de los resultados, fueron utilizados el software Microsoft Excel contenido en el paquete Microsoft Office 2016 y el Statgraphics Centurion. Los resultados de fenoles totales y flavonoides fueron expresados como media \pm desviación estándar de cada lote y comparadas sus medias empleando el test de Mínimas y Máximas Diferencias Significativas de Tukey, auxiliados del software Statgraphics Centurion. En todos los casos se consideró un $95 \%$ de confianza.

\section{Resultados}

La composición química cualitativa para los cinco extractos mostró la presencia de los metabolitos monitoreados: fenoles totales, taninos y flavonoides. Ello condiciona la posibilidad de cuantificación y muestra que en las ambiente de cultivo en que creció la planta existían las condiciones bióticas y abióticas para la expresión metabólica de estos metabolitos secundarios. La confirmación de la presencia de estos metabolitos en el extracto blando, demuestra que el proceso de obtención del mismo no modificó la composición química cualitativa de las flores utilizadas como materia prima. Los resultados se muestran en la Tabla 1 . 


\section{ORANGE JOURNAL}

Determinación cualitativa de fenoles totales y flavonoides

Tabla 1.

Composición química cualitativa del extracto blando de C. officinalis L.

\begin{tabular}{|c|c|c|c|c|c|c|}
\hline Metabolitos & Ensayos & $\begin{array}{c}\text { Oct. } \\
\mathbf{2 0 1 7}\end{array}$ & $\begin{array}{c}\text { Nov. } \\
\mathbf{2 0 1 7}\end{array}$ & $\begin{array}{c}\text { Dic. } \\
\mathbf{2 0 1 7}\end{array}$ & $\begin{array}{c}\text { Ene. } \\
\mathbf{2 0 1 8}\end{array}$ & $\begin{array}{c}\text { Feb. } \\
\mathbf{2 0 1 8}\end{array}$ \\
\hline $\begin{array}{c}\text { Taninos y } \\
\text { fenoles }\end{array}$ & Cloruro férrico & ++ & ++ & ++ & ++ & ++ \\
\hline \multirow{2}{*}{ Flavonoides } & $\begin{array}{c}\text { Ácido sulfúrico } \\
\text { (conc.) }\end{array}$ & ++ & ++ & ++ & ++ & ++ \\
\cline { 2 - 7 } & Shinoda & ++ & ++ & ++ & ++ & ++ \\
\cline { 2 - 7 } & Álcalis & ++ & ++ & ++ & ++ & ++ \\
\cline { 2 - 7 } & Rosemheim & ++ & ++ & ++ & ++ & ++ \\
\hline
\end{tabular}

Leyenda: ++: reacción positiva a ensayo realizado.

Fuente: Elaborada por los autores.

En la Tabla 2 se recogen los datos de la concentración equivalente de ácido gálico y la concentración de fenoles totales en los extractos blandos de C. officinalis L, mientras que en la Tabla 3 se muestran aquellos relacionados con la concentración de flavonoides totales. Los resultados evidencian que todos los extractos contienen compuestos fenólicos y flavonólicos en cantidades significativas, permitiendo sostener las propiedades medicinales y farmacológicas conferidas a la planta.

\section{Cuantificación de fenoles totales}

Tabla 2.

Concentración de fenoles totales presentes en los extractos de C. officinalis $L$.

\begin{tabular}{|c|c|c|}
\hline LOTES & Eqv Ácido Gálico $\boldsymbol{\mu g} / \mathbf{m L}$ & Fenoles Totales $(\mathbf{m g A G / 0 , 1 g ~ d e l ~ E B ) ~}$ \\
\hline Oct. 2017 & $2,003 \pm 0,07$ & $10,01 \pm 0,36^{\mathrm{a}}$ \\
Nov. 2017 & $2,118 \pm 0,03$ & $10,59 \pm 0,14^{\mathrm{ab}}$ \\
\hline Dic. 2017 & $2,122 \pm 0,06$ & $10,61 \pm 0,31^{\mathrm{ab}}$ \\
Ene. 2018 & $2,145 \pm 0,07$ & $10,72 \pm 0,37^{\mathrm{ab}}$ \\
Feb. 2018 & $2,216 \pm 0,05$ & $11,08 \pm 0,23^{\mathrm{b}}$ \\
\hline
\end{tabular}

Leyenda: letras diferentes indican diferencias estadísticamente significativas para un nivel de confianza de $95,0 \%(\mathrm{p}<0,05)$, por el test de rangos múltiples de menores y mayores diferencias significativas de Tukey (HSD).

Fuente: Elaborada por los autores. 


\section{Cuantificación de flavonoides}

Tabla 3.

Concentración de flavonoides totales presentes en los extractos de C. officinalis $L$.

\begin{tabular}{|c|c|c|}
\hline LOTES & Eqv quercetina $(\boldsymbol{\mu g} / \mathbf{m L})$ & $\begin{array}{c}\text { Flavonoides Totales } \\
\text { (mgQuerc/0,1g del EB) }\end{array}$ \\
\hline Oct. 2017 & $0,841 \pm 0,08$ & $1,68 \pm 0,17^{\mathrm{a}}$ \\
\hline Nov. 2017 & $0,914 \pm 0,08$ & $1,83 \pm 0,15^{\mathrm{a}}$ \\
\hline Dic. 2017 & $0,983 \pm 0,06$ & $1,97 \pm 0,12^{\mathrm{a}}$ \\
\hline Ene. 2018 & $0,988 \pm 0,06$ & $1,98 \pm 0,13^{\mathrm{a}}$ \\
\hline Feb. 2018 & $1,015 \pm 0,07$ & $2,03 \pm 0,14^{\mathrm{a}}$ \\
\hline
\end{tabular}

Leyenda: letras iguales indican que no existen diferencias estadísticamente significativas para un nivel de confianza de $95,0 \%(\mathrm{p}<0,05)$, por el test de rangos múltiples de menores y mayores diferencias significativas de Tukey (HSD).

Fuente: Elaborada por los autores.

\section{Discusión}

Se consultaron en la bibliografía numerosos estudios que muestran la cuantificación de fenoles totales en extractos de flores de $\mathrm{C}$ officinalis. Ćetković y colaboradores reportaron en 2003 un total de $15,12 \mathrm{mg} / \mathrm{g}$ de fenoles en extractos metanólicos de caléndula, determinándose por cromatografía de capa fina (TLC) ácidos fenólicos como: ácidos clorogénico, caféico, cumárico y vinílico. Igualmente, Rigane et al. (2013) reportaron una concentración de fenoles de 109,27mg/g en un extracto metanólico de caléndula.

Babaee y colaboradores (2013) expresaron un total de compuestos fenólicos de $313,40 \pm 6,52 \mathrm{mg} / \mathrm{g}$ en extractos hidroalcohólicos de la planta, evaluados mediante ensayo clínico para ser aplicados como enjuague bucal a pacientes con mucositis oral inducida por radioterapia; por otra parte Farahpour en 2014 estimó la concentración de polifenoles en un extracto hidroalcohólico de flores de esta planta en 37.01 \pm 0.015 g GAE g-1. En otro estudio Rigane, Chtourou, Ben Mahmoud, Medhioub y Ammar (2015) reportan valores de $109.27,51.62$ y $26.5 \mathrm{mg}$ GAE g-1 peso seco en hidro-metanol.

El contenido de polifenoles de un extracto hidroalcohólico de los pétalos de Calendula officinalis también fue determinado por Hernández-Rosas y colaboradores en 2018. Este extracto mostró que el contenido total de estos metabolitos fue de $37.01 \pm 0.015 \mathrm{mg}$ GAe g-1, siendo la rutina la más abundante.

También Álvarez en 2019 realizó la determinación de fenoles totales por el método de Folin Ciocalteu en un extracto con menstruo etanólico y otro con propilenglicol de pétalos de flores de caléndula officinalis. Los resultados de los análisis del extracto con propilenglicol expresados en equivalente de ácido gálico dieron como valores promedio de $5.410 \pm 0.025 \mathrm{mg}$ de AG/ $\mathrm{kg}$ de pétalos de caléndula. En relación al extracto etanólico el promedio fue de $4.935 \pm 0.043 \mathrm{mg}$ de $\mathrm{AG} / \mathrm{kg}$ de pétalos de caléndula. 


\section{ORANGE JOURNAL}

Como se puede observar en estos estudios consultados, independientemente del menstruo utilizado siempre se obtienen cantidades significativas de fenoles totales, aunque estas cantidades son diferentes, lo que demuestra la variabilidad en cuanto al contenido de compuestos fenólicos en esta especie.

En nuestra investigación en los cinco lotes estudiados se obtuvo la presencia de fenoles, coincidiendo con lo planteado en la literatura que reporta que la caléndula puede ser cultivada en todo el año, no presentando diferencias significativas en cuanto a su composición de metabolitos secundarios (Lastra y Piquet, 1999). La cantidad de estos metabolitos para el extracto blando obtenido a partir de las flores colectadas en el mes de octubre del 2017, difiere estadísticamente con respecto a las recolectadas en el mes de febrero del 2018, lo que pudo deberse a que existe una variación en el contenido de metabolitos activos en matrices de origen natural, que puede ser influenciada por diversos factores que dependen de la naturaleza de la planta (droga utilizada, variación natural entre plantas, quimio-variedad); de los factores como la siembra, la cosecha, almacenamiento y de los factores tecnológicos, que se encuentran relacionados con los procesos de secado y extracción (Muñoz ,2014; Llauradó et al, 2020).

El análisis estadístico aplicado, mostrado también en la Tabla 2, corrobora lo planteado anteriormente, siendo significativamente diferentes las medias de la concentración de fenoles totales de los extractos que no comparten una misma letra, con un nivel de confianza de $95,0 \%(\mathrm{p}<0,05)$.

Los valores que se obtuvieron para flavonoides, en todos los casos, fueron menores que los obtenidos para el análisis de fenoles (1,68-2,03 mgQuerc/0,1g). Esto se encuentra dentro de lo esperado, puesto que los flavonoides representan un subgrupo de metabolitos dentro de los compuestos fenólicos.

En estudios consultados, se ha reportado un contenido de 0,88 y $0,33 \%$ de flavonoides totales en las flores y receptáculos de caléndula, respectivamente (Hadson, 1985); mientras que Coello (2012) reporta un total de $0,54 \%$ en su tesis de grado "Elaboración y control de calidad de gel cicatrizante de sábila (Aloe vera) y caléndula (Calendula officinalis)".

Otros autores han reportado concentraciones de 96,17QEmg/100mL (Butnariu y Coradini, 2012), 76,44mg/g (Rigane et al., 2013) y 5,13mg/g en extractos metanólicos de la planta, identificando flavonoides de interés farmacológico como rutina, quercetina y algunos glicósidos flavonóidicos no identificados (Ćetković et al., 2003), mientras que en extractos hidroalcohólicos se han reportado valores de 76,66 $\pm 23,24 \mathrm{mg} / \mathrm{g}$ (Babaee et al., 2013).

Mogrovejo en 2014 en su tesis "Determinación del efecto cicatrizante de un gel estandarizado de Calendula officinalis L. (Caléndula) en animales de experimentación" elaboró un extracto de las flores por percolación, obteniéndo un extracto glicólico al $20 \%$ con una concentración de quercetina de 6.300 mg.kg-1. Estudios más recientes de Gomes y colaboradores (2016) han planteado que el contenido de estos metabolitos en la caléndula puede variar de 0,52 a $0,79 \%$. 
En nuestra investigación se observa el ligero aumento en las concentraciones de flavonoides para los diferentes lotes analizados, al igual que ocurre para fenoles totales, lo que puede deberse a la influencia de agentes externos en el desarrollo de los metabolitos secundarios en la planta. Sin embargo, a pesar de este incremento, la comparación de varianzas revela que no existen diferencias estadísticamente significativas entre las muestras en estudio, según el test de rangos múltiples de menores y mayores diferencias significativas de Tukey (HSD). De acuerdo a este análisis podemos inferir que, independientemente del mes del año en que sea cultivada la planta, el contenido de flavonoides presente en las flores se mantendrá en concentraciones similares. Estos resultados obtenidos son de gran importancia dado que la quercetina es la base de los flavonoides glicósidos principalmente relacionados con actividad antiinflamatoria y cicatrizante (Muñoz, Morgan y Trujillo, 2015) y por tanto la determinación y cuantificación de estos metabolitos en un preparado fitoterapéutico avala la calidad y efectividad de la formulación.

\section{Conclusiones}

Se constató la presencia de fenoles y flavonoides en el extracto blando de Calendula officinalis L. La cuantificación química del extracto blando arrojó un contenido de 9,65 a $11,31 \mathrm{mgAG} / 0,1 \mathrm{~g}$ y $1,51 \mathrm{a} 2,17 \mathrm{mgQ} / 0,1 \mathrm{~g}$ de fenoles y flavonoides, respectivamente, lo que sugiere su posibilidad de empleo como principio activo en formulaciones para uso estomatológico.

\section{Referencias Bibliográficas}

Álvarez, Y.E. (2018). Determinación de fenoles totales y la capacidad antioxidante de los pétalos de Caléndula officinalis L. (Caléndula), (Tesis de grado), Universidad Privada Autónoma del Sur. Arequipa. Perú.

Amić, D., Davidović-Amić, D., Beslo, D., Rastija, V., Lučić, B., \& Trinajstić, N. (2007). SAR and QSAR of the antioxidant activity of flavonoids. Current Medicinal Chemistry, 14(7), 827-45.

Arora, D., Rani, A., \& Sharma, A. (2013) A review on phytochemistry and ethnopharmacological aspects of genus Calendula. University Institute of Pharmaceutical Sciences, Panjab University, Chandigarh, India, 7(14), 179-187.

Avalos, A., Pérez, E. (2009). Metabolismo secundario de las plantas. Reduca (Biología) Serie Fisiología Vegetal, 2(3), 119-45.

Ayuso, R., Torrent, J., \& López, J. (2004) Estomatitis protésica: puesta al día. RCOE, 9(6), 657-62.

Babaee, N., Moslemi, D., Khalipour, M., Vejdani, F., Moghadamnia, Y., Bijani, A,..., Moghadamnia. AA. (2013). Antioxidant capacity of Calendula officinalis flowers extract and prevention of radiation induced oropharyngeal mucositis in patients with head and neck cancers: a randomized controlled clinical study. DARU Journal of Pharmaceutical Sciences 21(1),18.

Butnariu, M, \& Coradini, C.Z. (2012). Evaluation of Biologically Active Compounds from Calendula officinalis Flowers using Spectrophotometry. Chemistry Central Journal, 6, 35 . 


\section{ORANGE JOURNAL}

Ćetković, G.S., Đilas, S.M., Čanadanović-Brunet, J.M., Tumbas, V.T. (2003). ThinLayer Chromatography analysis and cavenging activity of marigold (Calendula officinalis L.) extracts. Acta Periodica Technologica (Serbia and Montenegro) 34, $1-148$.

Coello, R.J. (2012). Elaboración y control de calidad de gel cicatrizante a base de sábila (Aloe vera) y caléndula (Calendula officinalis) (Tesis de grado). Riobamba, Ecuador.

Cong-Cong, X.U., Bing, W., Yi-Qiong, P., JianSheng, T.A.O., \& Tong, Z. (2017). Advances in extraction and analysis of phenolic compounds from plant materials. Chin J Nat Med.,15(10), 721-31.

Domínguez, L. (2009). Utilización de flores de calédula (calendulae flos) en salsa de Carnes. (Tesis de Especialidad). Universidad Nacional de Colombia, Bogotá.

Domínguez, L. (2012). Efecto de la aplicación del extracto hidroalcohólico de flores de caléndula (Calendula officinalis) en la estabilizacion del color y vida útil en pulpa de frutas. (Tesis de Maestría). Universidad Nacional de Colombia, Bogota.

Escalona, J.C. (2005). Estudio químico farmacéutico de las hojas de Tamarindus indica L. Valoración teórica de la actividad de los flavonoides. (Tesis de maestría). ISCM, Santiago de Cuba.

Farahpour, M. R. (2014). Antioxidant activity, Antinociceptive and anti-inflammatory effects of Pot marigold hydroalcoholic extract on experimental animals. Inter. J. Pharm.Tech. Res., 1(974-4304-1),1640-1646.

Ferreira, I. C. F. R., Vaz, J. a, Vasconcelos, M. H., \& Martins, A. (2010). Compounds from wild mushrooms with antitumor potential. Anti-Cancer Agents in Medicinal Chemistry, 10, 424-436.

Gomes, I.C, Giardini, F.P, Montoya, S, Dias, V.V, Viana, J.P, \& Cecon, P.R. (2016). Growth, development and content of flavonoids in calendula (Calendula officinalis L.). Acta Scientiarum Agronomy, 38(1), 69-75

Hadson, V. (1985). Qualitative and quantitative analysis of flavonoids in plant products of cosmetic interest. Clujul Medical, 58(4), 378-81.

Hernández-Rosas, N.A. et al. (2018). Polyphenols antioxidant capacity, in vitro cytotoxic effect, human cancer cell lines of a hydroalcoholic extract from Calendula officinalis 1. Petals. Revista Especializada en Ciencias Químico-Biológicas, 21(1), 54-64. DOI: 10.22201/fesz.23958723e.2018.0.150

Lastra, H., \& Piquet, R. (1999) Calendula officinalis. Rev Cubana Farm., 33(3), 188-194.

Llauradó, G., Méndez, D., Hendrix, S., Escalona-Arranz, J.C., Fung, Y., Ochoa, A., García, J., Morris, H.J., Ferrer, A., Isaac, E., Beenaerts, N., Méndez, I., Orberá, T., Cos, P., \& Cuypers, A. (2020) .Antioxidants in Plants: A Valorization Potential Emphasizing the Need for the Conservation of Plant Biodiversity in Cuba. Antioxidants, 9, 1048. https://doi.org/10.3390/antiox9111048

Ministerio de Salud Pública MINSAP (2014). Formulario Nacional de Fitofármacos y Apifármacos. La Habana, Cuba: Centro para el Desarrollo de la Farmacoepidemiología.

Ministerio de Salud Pública MINSAP (1991). Medicamentos de origen vegetal. Extractos fluidos y Tinturas. Procesos tecnológicos. Norma Ramal de Salud Pública 311 (NRSP 311). La Habana, Cuba. 
Mogrovejo, A C. (2014). Determinación del efecto cicatrizante de un gel estandarizado de Calendula officinalis L. (Caléndula) en animales de experimentación. (Tesis de grado), Universidad Catolica de Santa Maria. AREQUIPA - PERU.

Muley, B, Khadabadi, S, \& Banarase, N. (2009). Phytochemical Constituents and Pharmacological Activities of Caléndula officinalis Linn (Asteraceae). Tropical Journal of Pharmaceutical Research, 8(5), p. 455-465.

Muñoz, J A. (2014) . Cuantificación de flavonoides, expresados como quercetina total, en algunos extractos y soluciones de uso oral a base de Calendula officinalis. (Tesis de Maestría), Universidad Nacional de Colombia. Facultad de Ciencias, Departamento de Farmacia Bogotá D.C., Colombia.

Muñoz, J A., Morgan, J E., \& Trujillo, M. (2015).Validation of an HPLC method for quantification of total quercetin in Calendula officinalis extracts. Revista Cubana de Farmacia, 49 (1), 91-102

Neukirch, H., D'Ambrosio, M., Via, J.,\& Guerriero, A. (2004). Simultaneous quantitative determination of eight triterpenoid monoesters from flowers of 10 varieties of Calendula officinalis L. and characterisation of a new triterpenoid monoester. Phytochem. Anal. 15, p. 30-35.

Ochoa, A., López, T., \& Colombat, M. (1999). Folleto para las Actividades Prácticas de las Asignaturas Farmacognosia y Química de los Productos Naturales. Santiago de Cuba.

Ogunleye, D.S., \& Ibitoye, S.F. (2003). Studies of antimicrobial activity and chemical constituents of Ximenia americana. Trop J Pharm Res, 2(2), 239-41.

Paolini, J, Barboni, T, Desjobert, JM, Djabou, N, Muselli, A, \& Costa, J. (2010). Chemical composition, intraspecies variation and seasonal variation in essential oils of Calendula arvensis L. Francia. Biochem. Syst. Ecol.,38(5), 865-74.

Pérez, G. (2003). Los flavonoides: antioxidantes o prooxidantes. Rev Cubana Invest Biomed, 22(1), 48-57.

Rigane, G., Ben Younes, S., Ghazghazi, H., \& Ben Salem, R. (2013). Investigation into the biological activities and chemical composition of Calendula officinalis L. growing in Tunisia. International Food Research Journal, 20(6): 3001-7.

Rigane, H., Chtourou, M., Ben Mahmoud, I., Medhioub, K. \& Ammar, E. (2015). Polyphenolic compounds progress during olive mill wastewater sludge and poultry manure co-composting, and humic substances building (Southeastern Tunisia). Waste Manag. Res., 33(1), 73-80. DOI: 10.1177/0734242X14559594.

Roig, J.T. (1991). Plantas medicinales, aromáticas o venenosas de Cuba. La Habana, Cuba: Editorial Científico Técnica.

Rotta, E. M., Windson, C., Haminiuk, I., Maldaner, L. \& Vissentainer, J. (2017). Determination of antioxidant activity and phenolic compounds of Muntingia calabura Linn peel by HPLC-DAD and UPLC-ESI-MS/MS. Int. J. Food Sci. Technol., 52, 954-963. DOI: 10.1111/ijfs.13359.

Valencia, E., Figueroa, I.I., Sosa, E., Bartolomé, M.C., Martínez, H.E. y García, M.E. (2017). Polifenoles: propiedades antioxidantes y toxicológica. Revista de la Facultad de Ciencias Químicas 16, 15-29.

WHO. (2013). Estrategia de la OMS sobre medicina tradicional 2014-2023. https://apps.who.int/iris/bitstream/handle/10665/95008/9789243506098_spa.pdf; jsessionid $=\mathrm{C} 159096994641 \mathrm{~F} 792 \mathrm{DAD} 23 \mathrm{EAD} 5 \mathrm{FF} 739 \mathrm{~A}$ ? sequence $=1$ 


\section{ORANGE JOURNAL}

Yassen, A.A., Habib, A.M., Zaghloul, S.M. \& Khaled, S.M. (2010). Effect of different sources of potassium fertilizers on growth yield, and chemical composition of Calendula officinalis. J. Amer. Sci., 6(12), 1044-1048 